\title{
Diversity of cell-mediated adhesions in breast cancer spheroids
}

\author{
ANDREA IVASCU and MANFRED KUBBIES \\ Roche Pharmaceutical Research Oncology, Penzberg, Germany
}

Received June 21, 2007; Accepted August 1, 2007

\begin{abstract}
Due to their three dimensional (3D) architecture, multicellular tumor spheroids mimic avascular tumor areas comprising the establishment of diffusion gradients, reduced proliferation rates and increased drug resistance. We have shown recently that the spontaneous formation of spheroids is restricted to a limited number of cell lines whereas the majority grow only as aggregates of cells with loose cellcell contacts when cultured in 3D. However, by the addition of reconstituted basement membrane ( $\mathrm{rBM}$, Matrigel ${ }^{\mathrm{TM}}$ ), aggregates can be transformed into spheroids with diffusion barriers and development of quiescent therapy-resistant cells. In this report, we investigated adhesion molecules responsible for rBM-driven versus spontaneous spheroid formation in a diverse population of eight breast tumor cell lines relevant for in vitro and in vivo antitumor drug testing. Inhibition of spheroid formation was monitored in the presence of adhesion molecule functional blocking antibodies and after siRNAmediated down-regulation of $\mathrm{E}$ - and $\mathrm{N}$-cadherin and integrin $\beta 1$ adhesion receptors. We identified that E-cadherin mediates the spontaneous formation of spheroids in MCF7, BT-474, T-47D and MDA-MB-361 cells, whereas N-cadherin is responsible for tight packing of MDA-MB-435S cells. In contrast, the matrix protein-induced transformation of 3D aggregates into spheroids in MDA-MB-231 and SK-BR-3 cells is mediated primarily by the collagen $\mathrm{I} /$ integrin $B 1$ interaction with no cadherin involvement. A combination of both, homophilic E-cadherin and integrin $31 /$ collagen I interaction establishes spheroids in MDA-MB-468 cells. These findings indicate that an evolutionary diverse and complex pattern of interacting cell surface proteins exists in breast cancer cells that determines the 3D growth characteristic in vitro, thereby influencing small molecule or antibody permeation in preclinical in vitro and in vivo tumor models.
\end{abstract}

\section{Introduction}

Preclinical in vitro cell models used for the development of new anti-cancer drugs have limited predictivity for in vivo

Correspondence to: Dr Manfred Kubbies, Pharmaceutical Research Oncology, Roche Diagnostics GmbH, Nonnenwald 2, 82377 Penzberg, Germany

E-mail: manfred.kubbies@roche.com

Key words: breast cancer, tumor spheroids, integrin, cadherin efficacy. This becomes especially evident using monolayer high-throughput cell culture technologies to screen for novel antineoplastic drugs (1). Since tumors establish drug gradients in vivo through molecule diffusion from blood vessels or through drug clearance, a limited drug exposure in these regions and the presence of quiescent cells and hypoxic acidic regions in tumors account for the insensitivity to many chemotherapeutic drugs (2).

The multicellular tumor spheroid model is frequently used to study anti-tumor drug effector functions since it mimics the in vivo avascular tumor architecture and physiology more closely compared to the monolayer cell model $(3,4)$. The three dimensional architecture of the spheroid model allows the analysis of cytotoxic drug effects in the context of a) diffusion gradients, b) adhesion and tight junction barriers and c) a heterogeneous tumor cell population of proliferating cells in the rim and non-cycling or dead cells in the core region of the spheroid (5-8). The multicellular clinical drug resistance that emerges from the so called 'contact effect' can be more closely simulated in spheroids. The enhanced cell-cell contacts of cells incorporated in spheroids or the interaction of cells with extracellular matrix (ECM) proteins can confer insensitivity to cytotoxic stimuli, since disruption of adhesions mediated by E-cadherin or by the interaction of cellular integrin receptors with hyaluronic acid, can sensitize cells in spheroids to chemotherapeutic treatment $(9,10)$.

In order to enable the large scale use of three dimensional cultures in high-throughput screening systems, we previously established a method to generate spheroids in a 96-well format within $24 \mathrm{~h}$ (11). The generation of one spheroid per well, with equal size and homogenous round geometry, ensures reproducible analysis since identical radial diffusion barriers towards the centre of the spheroid establish a similar microenvironment in each spheroid. A similar adhesion and tight junction protein expression is observed in rapidly compacted cells in spheroids by centrifugation compared to spheroids growing slowly from a small number of cells. However, many tumor cell lines establish only weak intercellular interactions forming aggregates with less tight associated cells (6), a property that does not represent compact tumor packaging, which alters drug penetration properties (12). With the addition of Matrigel ${ }^{\mathrm{TM}}$ these aggregates are transformed into spheroids in suspension which resemble more closely the morphology and diffusion properties of xenografts derived from these tumor cell lines (11).

It was previously shown that spheroids from different tumor tissues establish a spheroid-specific size-dependent onset of necrosis indicating that cell death depends on intrinsic, 
epigenetic properties of the tumor cells (6). We observed that in a panel of eight breast cancer tumor spheroids, the emergence of cell death was different between cell lines (11). However, spontaneously forming spheroids displayed a higher percentage of dead cells compared to aggregate forming cells, indicating that tightness of compaction has a significant effect on the development of a biochemical spheroid microenvironment resembling an avascular tumor area.

Previous studies described that E-cadherin-mediated cellcell adhesion dictates spontaneous formation of spheroids from colon and renal tumor cell lines (13-15). In some undifferentiated epithelial carcinomas loss of expression or dysfunction of the cell-cell adhesion molecule E-cadherin is observed, which results in abrogation of spheroid formation in these tumor cell lines $(16,17)$. Moreover, the interaction of cells with extracellular matrix proteins can also lead to the formation of spheroids. For example, integrin $\beta 1$-fibronectin interaction has been shown to be involved in spheroid formation from ovarian tumor cells (18), and expression of the integrin $\alpha 5 \beta 1$ fibronectin receptor in $\mathrm{CHO}$ cells conferred strong tissue cohesion in $3 \mathrm{D}$ cultures of CHO cells in the presence of fibronectin (19). Our finding that $\mathrm{rBM}$ increases aggregate compaction in many ontogenetic different tumor cell lines also suggests that the interaction of extracellular proteins with their ligands might be involved in spheroid formation (11). A loss or aberrant expression of adhesion molecules has been identified in tumor cells thereby altering their aggregation properties in vitro (20-23).

The aim of this study was to identify the cellular adhesion molecules involved in spontaneous and extracellular matrix driven spheroid formation in eight breast cancer cell lines. Cell-cell interacting E- and N-cadherin as well as the cellmatrix interacting receptor integrin $B 1$ were investigated using siRNA and inhibitory antibodies. Our results indicate that in the majority of the cell lines either a single cadherin or ECM-integrin receptor interaction induces tight cell packaging in breast cancer spheroids, and that only in MDA-MB-468 cells both E-cadherin plus integrin $ß 1$ contribute to spheroid formation.

\section{Materials and methods}

Cell culture. Eight breast cancer cell lines were investigated: MDA-MB-231 (ATCC HTB-26), MDA-MB-468 (ATCC HTB-132), MDA-MB-435S (ATCC HTB-129), MDA-MB361 (ATCC HTB-27), SK-BR-3 (ATCC HTB-30), T-47D (ATCC HTB-133), MCF7 (ATCC HTB-22) and BT-474 (ATCC HTB-20). All cell lines except BT-474 and SK-BR-3 were cultured in RPMI-1640 (PAA Laboratories GmbH, Innsbruck) supplemented with $10 \%$ FCS (Gibco-BRL, Karlsruhe) and $2 \mathrm{mM}$ L-Glutamine (Gibco-BRL). SK-BR-3 cells were cultivated in McCoy's 5A (Gibco-BRL) with the same additives; for BT-474 cells $1 \mathrm{mM}$ sodium pyruvate (Gibco-BRL) and $1 \mathrm{mM}$ HEPES (Sigma-Aldrich, Deisenhofen) were added to the RPMI culture medium. For optimal spheroid formation of MDA-MB-231, MDA-MB-468, MDA-MB-435S and SK-BR-3 cells, 2.5\% rBM (Matrigel, BD Biosciences, Bedford, MA) was added to the medium.

Spheroid generation. Monolayer cells were detached with Accutase (PAA Laboratories $\mathrm{GmbH}$, Innsbruck) to generate a single cell suspension. The cell number was determined using a CASY instrument (Schärfe-Systems, Reutlingen), and the cell suspension was diluted in ice-cold medium to $2.5 \times 10^{4}$ cells $/ \mathrm{ml}$. The rBM was thawed on ice overnight and added at a final concentration of $2.5 \%$ with ice-cold pipette tips to the cell suspension. A volume of $200 \mu 1$ (=5000 cells) of the cell suspension was added to each well of a 96-well plate with a round (Corning Inc., New York) or conical (Nunc, Roskilde, Denmark) bottom. To prevent cell attachment the plates were precoated with $50 \mu 10.5 \%$ poly-HEMA (Polysciences, Eppelheim) in $95 \%$ ethanol and air dried at $37^{\circ} \mathrm{C}$ for three days. The spheroid formation was initiated by centrifugation of the plates at $1000 \mathrm{x}$ g for 10 min (minutes) using an Eppendorf 5810 centrifuge (Eppendorf AG, Hamburg) with swinging buckets. The plates were incubated under standard cell culture conditions at $37^{\circ} \mathrm{C}$ and $7 \% \mathrm{CO}_{2}$ in humidified incubators. The incorporation of ECM components was investigated with fluorescent labelled matrix components which were added to the wells prior to centrifugation: $5 \mu \mathrm{g} / \mathrm{ml}$ of collagen I-FITC (Molecular Probes, Oregon, USA), collagen IV-Oregon Green 488 (Molecular Probes) or fibronectin-rhodamine (Cytoskeleton, Denver, USA).

Transfection of siRNA molecules. The breast cancer cell lines were seeded between $10-30 \%$ confluency into 6-well plates and allowed to attach for $24 \mathrm{~h}$. Prior to integrin $\beta 1$ siRNA transfection cells were washed once with serum-free OptiMEM medium (Gibco-BRL). Oligofectamine (Invitrogen, Carlsbad, CA, USA) was used to transfect $100 \mathrm{nM}$ siRNA duplexes in serum-free medium. After $4 \mathrm{~h}$ of incubation the serum concentration was adjusted to $10 \%$ in OptiMEM medium and cells were incubated for a further $72 \mathrm{~h}$ until analysis. The siRNA molecules were purchased as duplexes from Dharmacon Research (Lafayette, Colorado, USA). Integrin ß1 (Int ß1): 5'-PUCAUUCAUCAGAUCUGUUCUU-3' and luciferase (luc) (as unspecific control): 5'-NNCGUACGCGG AAUACUUCGA-3'. The relative amount of integrin 31 mRNA in the samples transfected with the relevant siRNA was calculated using the following formula $100 / 2^{\Delta \mathrm{cp}}[\Delta \mathrm{cp}$ (crossing point $)=c p$ int $B 1-c p$ luc) and normalized to the cell line with the highest integrin $B 1$ mRNA quantity, namely MDAMB-231.

Inhibitory antibodies. Adhesion molecule inhibitory antibodies were added at $10 \mu \mathrm{g} / \mathrm{ml}$ to the well prior to centrifugation of the cell-containing 96-well plate (see spheroid generation). The inhibitory antibodies applied were anti-E-cadherin clone SHE78-7 (Takara, Japan), anti-integrin B1 clone P5D2 (Chemicon International, Temecula, CA, USA) and anti-Ncadherin clone CG-4 (Sigma-Aldrich). The spheroid morphology was assessed after a 3-, 24-, 48- and 72-h culture period.

$R T$-PCR. Cells from a single well of a 6-well plate were lysed $72 \mathrm{~h}$ after siRNA transfection and RNA was isolated with the RNeasy kit (Qiagen, Hilden). Reverse transcription and real-time PCR were performed as single tube reactions using the LightCycler RNA master SYBR-Green kit (Roche Diagnostics, Mannheim). RNA template (250 ng), $0.5 \mu \mathrm{M}$ primers detecting integrin $\beta 1,3.25 \mathrm{nM} \mathrm{Mn}(\mathrm{OAc})_{2}$ and $7.5 \mu 1$ enzyme (RNA master SYBR-Green I) were mixed to a total 
volume of $20 \mu 1$ according to the manufacturer's protocol. The reactions were run in a LightCycler instrument (Roche Diagnostics) with the following parameters: RT at $61^{\circ} \mathrm{C}$ for $20 \mathrm{~min}$ and PCR with 40 cycles at $95^{\circ} \mathrm{C}$ for $5 \mathrm{sec}, 52^{\circ} \mathrm{C}$ for $5 \mathrm{sec}, 72^{\circ} \mathrm{C}$ for $20 \mathrm{sec}$ and fluorescence recording at $70^{\circ} \mathrm{C}$. In order to standardize the amount of mRNA used for integrin B1 quantification, the mRNA expression of the housekeeping enzyme, GAPDH was also detected. The same parameters were used for the RT-PCR, except a change in primer annealing temperature $\left(57^{\circ} \mathrm{C}\right.$ for $\left.5 \mathrm{sec}\right)$ and fluorescence recording at $76^{\circ} \mathrm{C}$. The primer molecules were designed using the Vector NTI 9.1 software (Invitrogen) and purchased from Metabion Int. (Martinsried, Germany). The primer sequences applied were as follows: integrin $\beta 1$ forward, 5'-CGAGGTCATGGT TCATGTTG-3' and reverse, 5'-CCCATTTGGCATTCATTT TC-3'; GAPDH forward, 5'-GAAATCCCATCACCATCTTC C-3' and reverse, 5'-ATGAGTCCTTCCACGATACC-3'. The integrin $\beta 1$ mRNA amount in each sample was determined by analysis of the crossing points of the RT-PCR using the LightCycler 3.5 software. The crossing points were determined from two independent PCR reactions and the level of integrin ß1 mRNA was related to the level of GAPDH mRNA detected in the same sample. The crossing points were analysed in three independent experiments.

Flow cytometry. Single cell suspensions were generated from monolayer and spheroid cultures by incubation and resuspension with cold PBS/5 mM EDTA. The cell number was adjusted to $5 \times 10^{5}$ cells per tube. The entire staining procedure was performed on ice. The cells were centrifuged and resuspended in $100 \mu \mathrm{PBS} / 2 \%$ FCS. For cell surface expression analysis $2.5 \mu \mathrm{g} / \mathrm{ml}$ anti-E-cadherin clone HECD-1 (Zymed, San Francisco), anti-integrin 31 clone 4B7 (Oncogene, San Diego), anti-N-cadherin clone CG-4 (Sigma-Aldrich) or antimouse IgG1 isotype (BD Biosciences, San Jose, USA) were added to the suspension. After a 30-min incubation at $4^{\circ} \mathrm{C}$, the cells were washed once with $\mathrm{PBS} / 2 \% \mathrm{FCS}$ and incubated for an additional $30 \mathrm{~min}$ at $4^{\circ} \mathrm{C}$ in $100 \mu \mathrm{lPBS} / 2 \% \mathrm{FCS}$ containing $5 \mu \mathrm{g} / \mathrm{ml}$ anti-mouse Alexa 488 antibody (Molecular Probes). Thereafter, cells were washed once, resuspended in $500 \mu 1 \mathrm{PBS} / 2 \%$ FCS and the immunofluorescence was analysed by flow cytometry (FACScan, Becton Dickinson, San Jose). The intensity of the positive cell populations was calculated as increase of the mean of the specific antibody sample compared the isotype antibody control sample (Cell Quest Version 3.3 software).

Apoptosis assessment. Spheroids were generated using 5000 cells per spheroid and 2.5\% rBM for MDA-MB-435S, MDAMB-468, MDA-MB-231 and SK-BR-3 cells. Functional blocking antibodies were added at $5 \mu \mathrm{g} / \mathrm{ml}$ prior to centrifugation. Centrifuged plates were incubated at $37^{\circ} \mathrm{C}$ for $48 \mathrm{~h}$ prior to analysis. For apoptosis analysis, eight spheroids from identical incubation conditions were pooled, washed once with phosphate-buffered saline (PBS), resuspended in Accutase solution for dissociation. The single-cell suspensions were stained with annexin- $\mathrm{V}$-fluos and propidium iodide in the presence of supplemented $2 \mathrm{mM} \mathrm{CaCl}{ }_{2}$ (annexin-V-fluos staining kit; Roche Diagnostics $\mathrm{GmbH}$ ). The fluorescence from 10000 cells was acquired using a fluorescence-activated
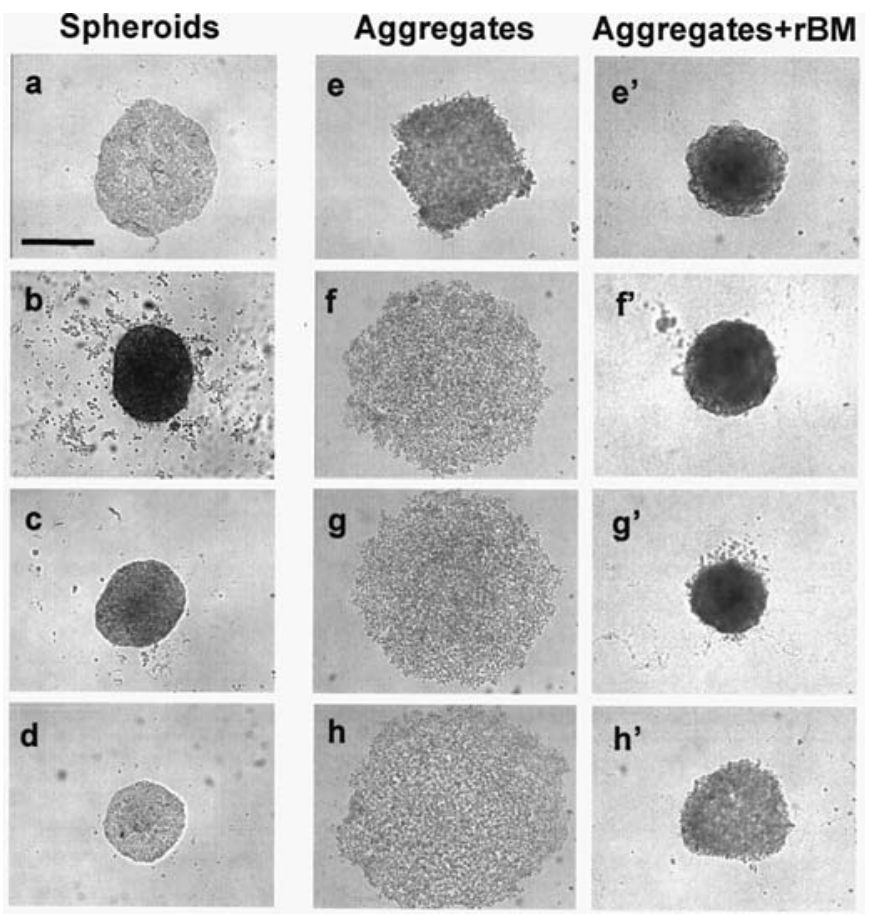

Figure 1. Spheroid morphology of eight breast cancer lines. Spheroids and aggregates were generated from 5000 cells in polyHEMA-coated 96 wells and cultured for $24 \mathrm{~h}$. Compact spheroids: MCF7 (a), BT-474 (b), T-47D (c), MDA-MB-361(d). Tight aggregates: MDA-MB-435S (e). Loose aggregates: MDA-MB-231 (f), MDA-MB-468 (g), SK-BR-3 (h). Compaction of aggregates into spheroids was achieved by the addition of $2.5 \% \mathrm{rBM}$ prior to centrifugation (e'-h'). Bar: $500 \mu \mathrm{m}$.

cell sorting (FACS) scan instrument (Becton Dickinson, San Jose, CA). Quadrant statistics was applied on the dot plots, with the number of viable cells located in the lower-left quadrant.

Microscopy. The morphology of the spheroids was assessed and recorded using an Axiovert 135 Microscope (Zeiss, Jena) and a $x 5$ objective. Pictures were taken with a Xillix Microimager Camera (Xillix Techn. Corp., Richmond, Canada) using the Open Lab 2.2.5 Software (Improvision, UK). The size of the spheroids was analysed using the calibration software analysis tools and an objective calibration slide (Zeiss).

\section{Results}

3D morphology of breast cancer cell lines. 3D suspension cultures of breast cancer cell lines can be grouped into three categories: compact spheroids, tight aggregates and loose association of cells. Four out of eight cell lines tested, MCF7, BT-474, T-47D and MDA-MB-361, spontaneously formed spheroids in suspension in the polyHEMA-coated 96-well plate within a $24-\mathrm{h}$ culture period. The spheroids were fully compact, with tight cell-cell adhesions where individual cell margins were barely detectable (Fig. 1a-d). The disintegration of these spheroids required extended trypsinization time and mechanical shearing. Previously MDA-MB-361 cells did not spontaneously form spheroids (11). Since the cell line used was claudin-1 transfected (24) and transdifferentiation events 
Table I. E-cadherin, N-cadherin and integrin $\beta 1$ expression on the cell surface of eight breast cancer cell lines grown for $24 \mathrm{~h}$ as a monolayer and as spheroids.

\begin{tabular}{|c|c|c|c|c|c|c|c|}
\hline \multirow[b]{2}{*}{ 3D morphology } & \multirow[b]{2}{*}{ Cell line } & \multicolumn{2}{|c|}{ E-cadherin } & \multicolumn{2}{|c|}{ N-cadherin } & \multicolumn{2}{|c|}{ Integrin $ß 1$} \\
\hline & & $2 \mathrm{D}$ & $3 \mathrm{D}$ & $2 \mathrm{D}$ & $3 \mathrm{D}$ & $2 \mathrm{D}$ & $3 \mathrm{D}$ \\
\hline \multirow[t]{4}{*}{ Compact spheroids } & MCF7 & 239 & 409 & 0 & 0 & 184 & 164 \\
\hline & ВТ-474 & 166 & 624 & 0 & 0 & 45 & 59 \\
\hline & $\mathrm{T}-47 \mathrm{D}$ & 211 & 645 & 0 & 0 & 222 & 154 \\
\hline & MDA-MB-361 & 181 & 328 & 0 & 0 & 165 & 138 \\
\hline Tight aggregates & MDA-MB-435S & 0 & 0 & 213 & 87 & 137 & 195 \\
\hline \multirow[t]{3}{*}{ Loose aggregates } & MDA-MB-231 & 0 & 0 & 0 & 0 & 241 & 652 \\
\hline & MDA-MB-468 & 120 & 313 & 0 & 0 & 62 & 92 \\
\hline & SK-BR-3 & 0 & 0 & 0 & 0 & 47 & 70 \\
\hline
\end{tabular}

Quantitative analysis of the mean fluorescence of E-cadherin and integrin B1 expression in breast cancer cell lines. The antibody fluorescence of viable cells was quantitated as increase over isotype control stained cells (channel numbers).

\section{Integrin ß1}
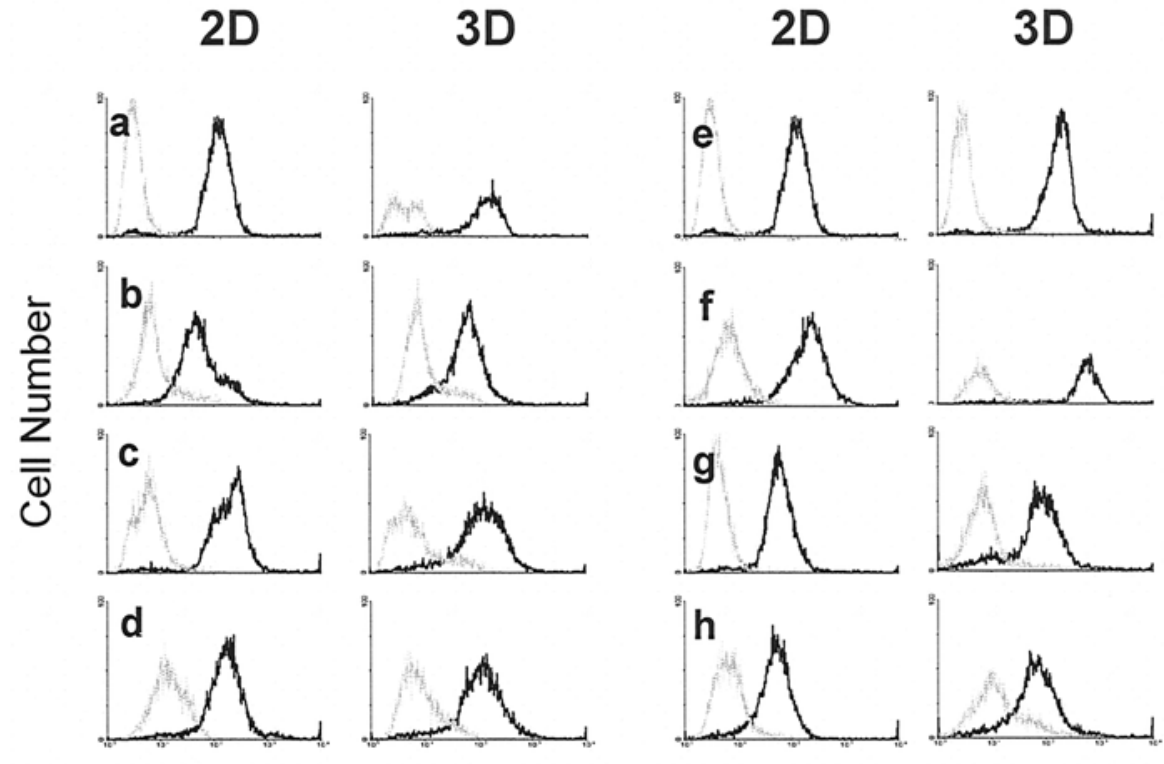

Fluorescence Intensity

Figure 2. Cell surface expression of integrin $\beta 1$ in eight breast cancer cell lines grown as monolayer culture (2D) and as spheroids (3D). Flow cytometric analysis of eight breast cancer cell lines stained with isotype control (dotted lines) and integrin ß1-specific antibody (solid lines). MCF7 (a), BT-474 (b), T-47D (c), MDA-MB-361 (d), MDA-MB-435S (e), MDA-MB-231 (f), MDA-MB-468 (g) and SK-BR-3 (h). Aggregates were compacted into spheroids with $2.5 \% \mathrm{rBM}(\mathrm{e}-\mathrm{h})$.

due to clonal selection could not be excluded, we repeated the morphology assessment with a non-transfected MDAMB-361 cell line.

In contrast, MDA-MB-435S cells formed aggregates of cells when cultured in 3D within a 24-h culture time (Fig. 1e). Extended cultivation increased the tightness of these aggregates with spotted sides of compaction but a full compaction was not achieved. The aggregates were easily disintegrated by short trypsin incubation and low mechanical force. Even more loose aggregates were generated from MDA-MB-231, MDAMB-468 and SK-BR-3 cells (Fig. 1f-h). Gentle agitation of these aggregates disturbed the loose cell-cell contacts giving rise to single cell suspensions. The addition of $2.5 \% \mathrm{rBM}$ to the aggregate forming cells prior to centrifugation tightened the cell-cell contacts and induced the formation of compact spheroids with round geometry within $24 \mathrm{~h}$ of cultivation 


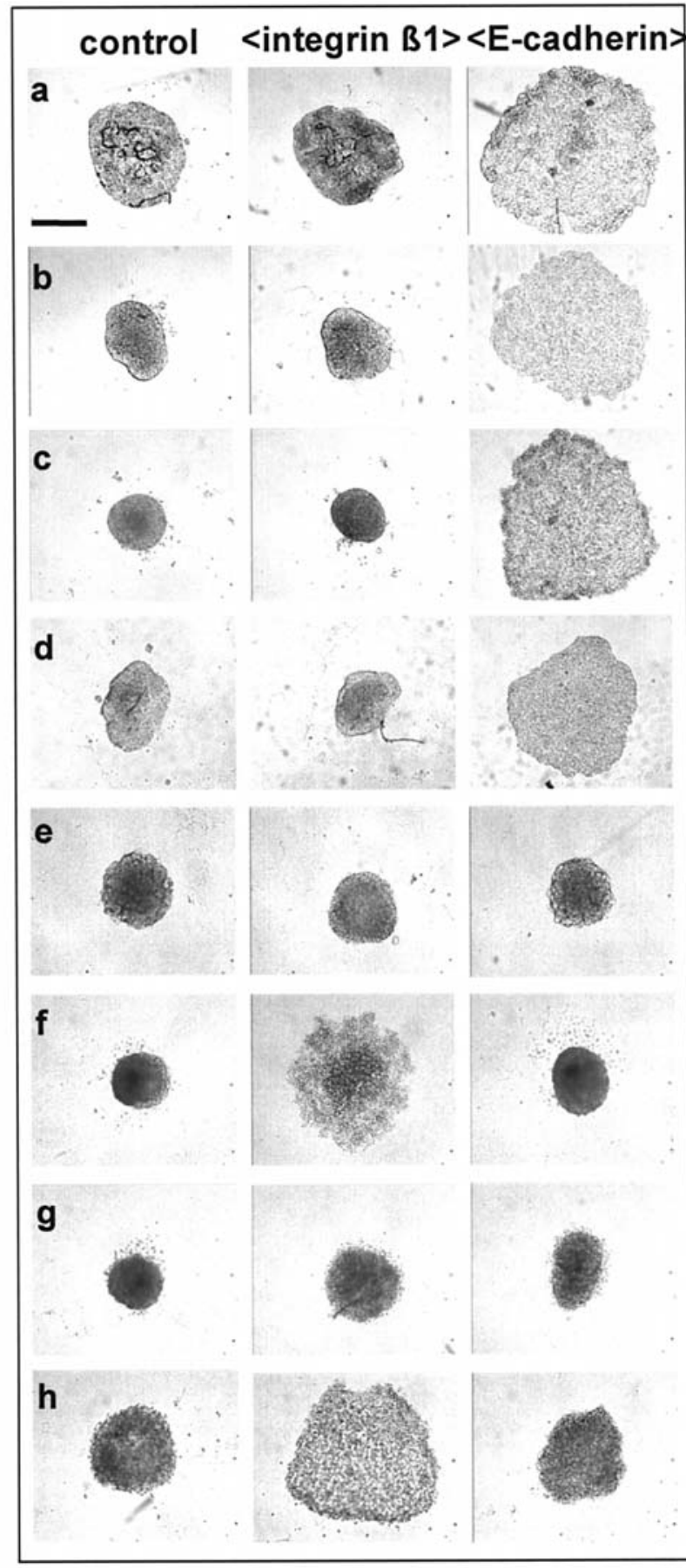

Figure 3. Inhibition of spheroid formation with integrin $B 1$ and E-cadherin functional blocking antibodies. Spheroid formation was performed with 5000 cells/well in the absence (left lane) or in the presence of inhibitory antibody (middle lane: integrin $\beta 1$ and right lane: E-cadherin) in 96-well plates followed by a subsequent cultivation period of $48 \mathrm{~h}$. MCF7 (a), BT-474 (b), T-47D (c), MDA-MB-361 (c), MDA-MB-435S (e), MDA-MB-231 (f), MDA-MB-468 (g), and SK-BR-3 (h). To enable spheroid formation $2.5 \%$ rBM was added to MDA-MB-435S, MDA-MB-231, MDA-MB-468 and SK-BR-3 cells. Bar: $500 \mu \mathrm{m}$.

(Fig. 1e'-h'). The addition of rBM to the four cell lines spontaneously forming spheroids, MCF7, BT-474, T-47D and MDA-MB-361 did not increase the spheroid tightness. In contrast, cells were able to attach to the bottom of the polyHEMA-coated plate and displayed a rather flat morphology (data not shown).
Cell surface expression of E-cadherin, $N$-cadherin and integrin $\beta 1$. E-cadherin, $\mathrm{N}$-cadherin and integrin $\beta 1$ cell surface receptor expression was comparatively analyzed in monolayer cells and spheroids in eight breast cancer cell lines applying immunostaining and FACS analysis of viable cells. As evident from Table I, five out of eight breast cancer cell lines expressed E-cadherin on the cell surface. MCF7, BT-474, T-47D and MDA-MB-361 cells expressed high amounts of E-cadherin whereas MDA-MB-468 cells expressed lower levels. The four E-cadherin high expressing cell lines spontaneously formed spheroids in vitro (Fig. 1a-d), whereas MDA-MB-468 cells exhibited a loose aggregate morphology in 3D (Fig. 1g). An increase of E-cadherin cell surface expression was observed in spheroids with up to three times more E-cadherin compared to monolayer cultures (Table I).

In contrast to E-cadherin, integrin $\beta 1$ was detected at significant levels on the cell surface of all cell lines tested (Fig. 2 and Table I). There was an approximate 5-fold difference of expression within the eight breast tumor cell lines, with MDA-MB-231 expressing the highest and BT-474 the lowest amount of integrin $B 1$ (Table I). There was no correlation between the expression levels of integrin $B 1$ and the 3D morphology of the breast tumor cell lines. However, aggregate forming cell lines displayed a higher expression of integrin $\beta 1$ in $\mathrm{rBM}$ generated spheroids compared to monolayer cultures (Table I). On the contrary, cell lines spontaneously forming spheroids displayed a slight decrease of integrin $B 1$ expression. $\mathrm{N}$-cadherin expression was found in MDA-MB$435 \mathrm{~S}$ cells only, with a lower expression in spheroids.

Inhibition of spheroid formation with inhibitory antibodies. To assess the involvement of E-cadherin homophilic interaction and integrin $\beta 1 /$ collagen interaction in spheroid formation, their physiological function was inhibited with blocking antibodies (bAB). Inhibition of the E-cadherin mediated cell-cell adhesion led to the disintegration of the spontaneously forming spheroids of MCF7, BT-474, T-47D and MDA-MB-361 cells (Fig. 3a-d, right lane). Disruption of the compact spheroids was not effective in inhibiting the integrin $\beta 1$ receptor (Fig. 3a-d, middle lane).

In contrast, the rBM-induced spheroids from MDA-MB435S, MDA-MB-231, MDA-MB-468 and SK-BR-3 cells were not inhibited by the E-cadherin inhibitory antibody (Fig. 3e-h, right lane). The inhibition of the integrin $B 1$ subunit suppressed the rBM-driven spheroid formation of MDA-MB-231 and SK-BR-3 cells (Fig. 3f and h, middle lane), but not of MDAMB-435S and MDA-MB-468 cells (Fig. 3e and g, middle lane). In MDA-MB-468 cells, the rBM-driven spheroid formation was disturbed only in the presence of both, Ecadherin and integrin $\beta 1$ blocking antibodies (Fig. 4Ac).

Previous studies showed that some breast tumor cell lines express $\mathrm{N}$-cadherin (25). Therefore we investigated an inhibitory antibody against this adhesion molecule on all breast tumor cell lines. Only the rBM-induced spheroid formation from MDA-MB-435S cells was abrogated in the presence of the $\mathrm{N}$-cadherin interaction blocking antibody (Fig. 4Bc). The spheroid morphology from the remaining seven breast tumor cell lines was not affected by this antibody (data not shown).

The dissociation of the spheroids after incubation with functional antibodies was not due to cell death since no 

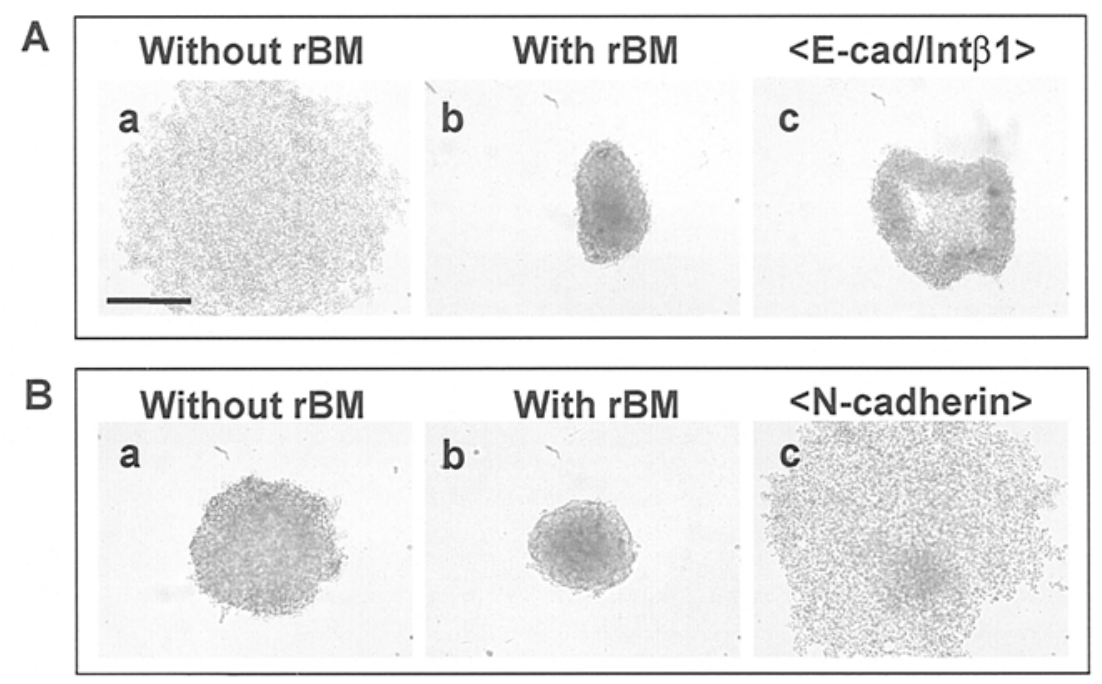

Figure 4. Inhibition of spheroid formation in MDA-MB-468 and MDA-MB-435S cells. Panel A: MDA-MB-468 cells were cultivated without rBM (a), with $\mathrm{rBM}$ (b) and with $\mathrm{rBM}$ in the presence of $5 \mu \mathrm{g} / \mathrm{ml}$ inhibitory antibodies against integrin $\mathrm{B} 1+$ E-cadherin (c). Panel B: MDA-MB-435S cells were cultivated without $\mathrm{rBM}(\mathrm{a})$, with $\mathrm{rBM}$ (b) and with $\mathrm{rBM}$ in the presence of $5 \mu \mathrm{g} / \mathrm{ml}$ inhibitory antibody against $\mathrm{N}$-cadherin. Spheroids and aggregates were generated from 5000 cells/well in 96-well plates and cultivated for $48 \mathrm{~h}$. Bar: $500 \mu \mathrm{m}$

Without rBM
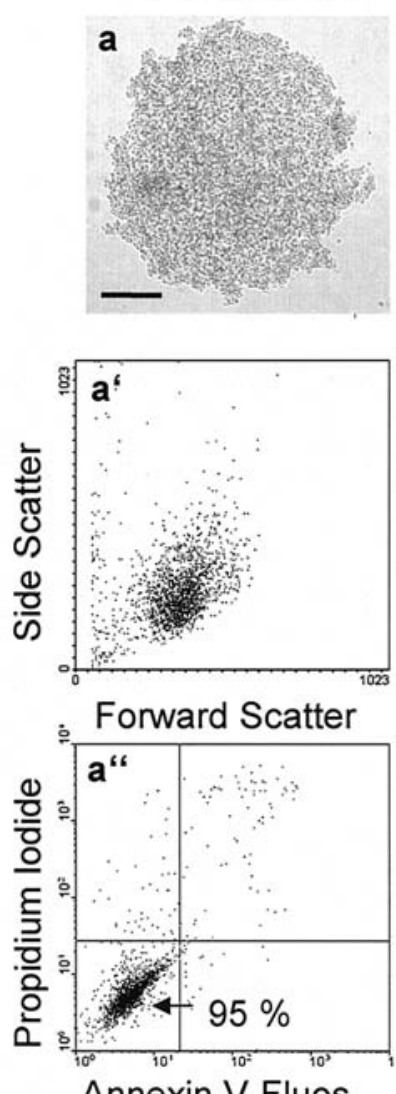

With rBM
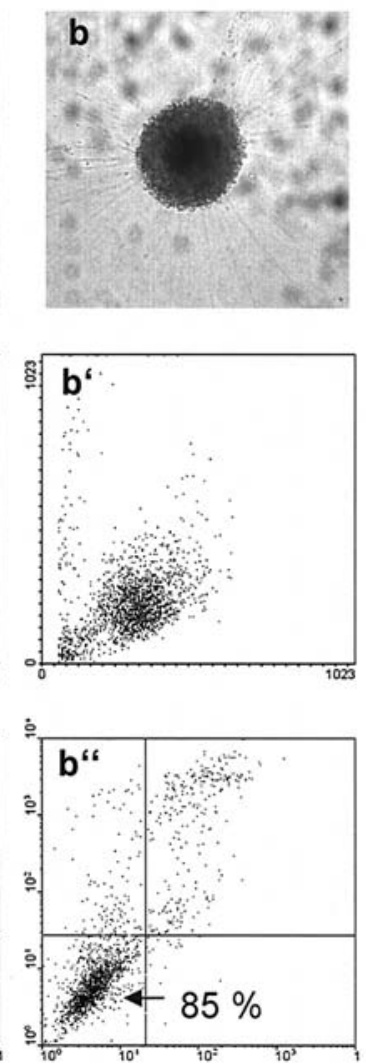

With rBM and $<\operatorname{lnt} \beta 1>$
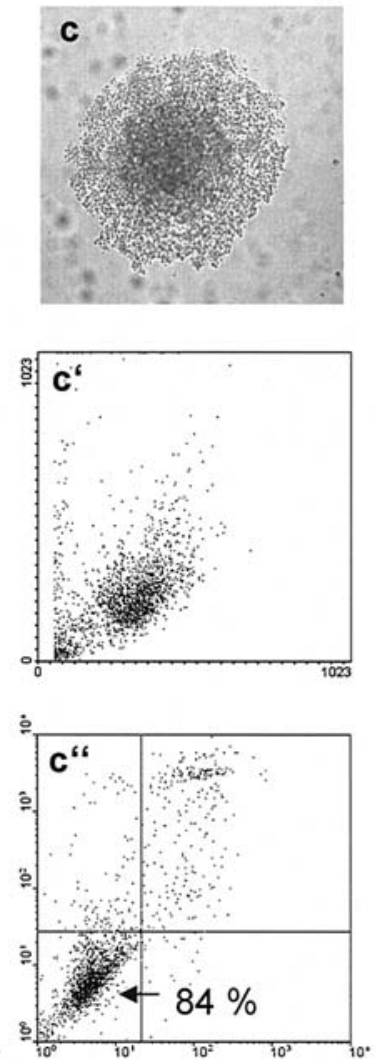

Figure 5. Apoptosis detection after integrin B1-mediated inhibition of spheroid formation in rBM-induced spheroids from MDA-MB-231 cells. Aggregate (a) and spheroid (b) formation from MDA-MB-231 cells in the absence and presence of rBM. Inhibition of rBM-induced spheroid formation after incubation with $10 \mu \mathrm{g} / \mathrm{ml}$ integrin $\mathrm{B} 1$ functional blocking antibody (c). The corresponding cell size and granularity are shown in a', $\mathrm{b}^{\prime}$ and $\mathrm{c}^{\prime}$, respectively. Apoptosis analysis of MDA-MB-231 3D cultures: without rBM (a"), with rBM (b") and with rBM in the presence of $10 \mu \mathrm{g} / \mathrm{ml}$ integrin $B 1$ functional blocking antibody (c"). Spheroids and aggregates were generated from 5000 cells/well in 96 -well plates and cultivated for $48 \mathrm{~h}$ prior to analysis. Bar: $500 \mu \mathrm{m}$.

change of cell viability was observed in annexinV-PI staining assays. Fig. 5 illustrates the dissociation of MDA-MB-231 spheroids generated by $\mathrm{rBM}$ addition in the presence of integrin $B 1$ blocking antibodies in correlation with cell death measurements. The compaction of MDA-MB-231 aggregates into spheroids paralleled the minor increase in cell death (10\%) 

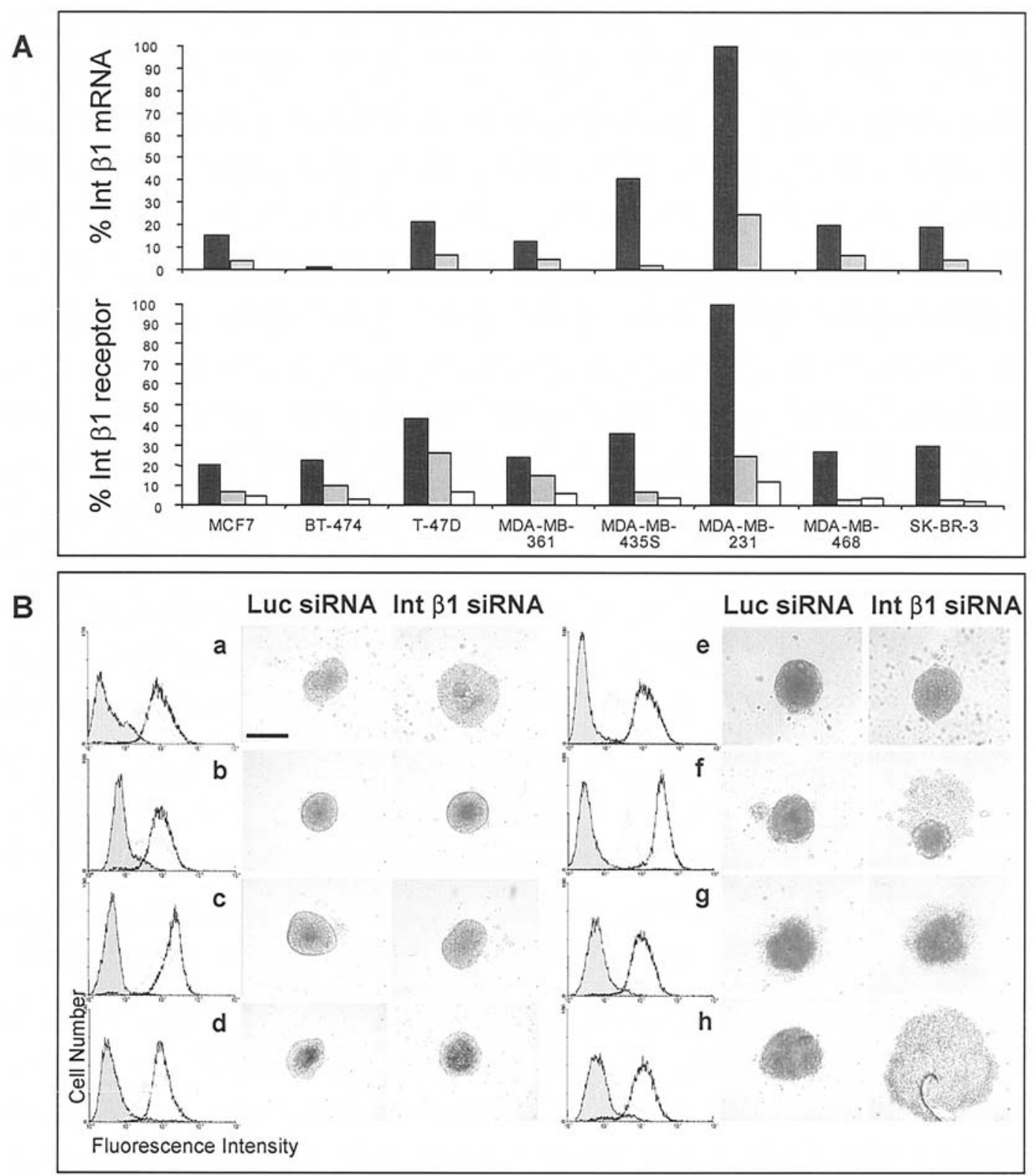

Figure 6. Alteration of spheroid morphology in breast cancer spheroids after siRNA-mediated down-regulation of integrin B1. Panel A: siRNA-dependent down-regulation of integrin $B 1$ mRNA (RT-PCR) and surface protein (FACS). mRNA and protein levels were normalized to 100\% level relative to MDAMB-231 cells (highest expression levels). Black bars represent integrin $\beta 1$ mRNA (upper panel) and protein (lower panel) expression of the total population after control siRNA transfection (luciferase). The grey bars represent the mRNA and protein amount after integrin $B 1$ siRNA transfection. The subpopulation with decreased receptor expression is represented by the white bars (lower panel). Panel B: FACS analysis of integrin B1 cell surface expression and corresponding spheroid morphology after siRNA transfection: isotype controls (grey fills), control siRNA (black line), integrin 31 siRNA (dotted line). Spheroid (a-d) and aggregate (e-h) forming breast cancer cell lines. Spheroids were formed from 5000 cells/well without rBM (a-d) and with $2.5 \% \mathrm{rBM}$ (e-h). MCF7 (a), BT-474 (b), T-47D (c), MDA-MB-361 (d), MDA-MB-435S (e), MDA-MB-231 (f), MDA-MB-468 (g) and SK-BR-3 (h). All analyses were performed $72 \mathrm{~h}$ post transfection. Bar: $500 \mu \mathrm{m}$.

(Fig. 5a, a", b and b") and the same viability was observed in rBM-induced MDA-MB-231 spheroids incubated with integrin ß1 blocking antibodies (Fig. 5c and $\mathrm{c}^{\prime}$ ). Also no change in morphology of MDA-MB-231 cells was observed after integrin B1 blockage as can be seen in the forward/side scatter dot blot (Fig. 5a', b' and c').

Inhibition of spheroid formation with integrin $\beta 1$ siRNA. Since antibodies might excert additional effector functions beyond sterical hindrance, the integrin $B 1$ inhibitory antibody results were confirmed by mRNA down-regulation of integrin B1 using siRNA. The morphology of 3D breast cancer cell cultures was evaluated by microscopic inspection and correlated to the alteration of the integrin $\beta 1$ cell surface expression after a 72-h monolayer siRNA treatment followed by a $24-\mathrm{h}$ 3D culture (Fig. 6). Of the luciferase control siRNA-transfected breast tumor cell lines, MDA-MB-231 cells expressed the highest mRNA amount of integrin $\beta 1$, whereas the other cell lines expressed $<50 \%$ of integrin $B 1$ mRNA (Fig. 6A, black bars, upper panel). The siRNA down-regulation of the integrin $\beta 1 \mathrm{mRNA}$ was very efficient $72 \mathrm{~h}$ post transfection, with all cell lines displaying a decrease in integrin $\beta 1$ mRNA of between $60 \%$ and $95 \%$ compared to the control transfection (Fig. 6A, grey bars, upper panel). 

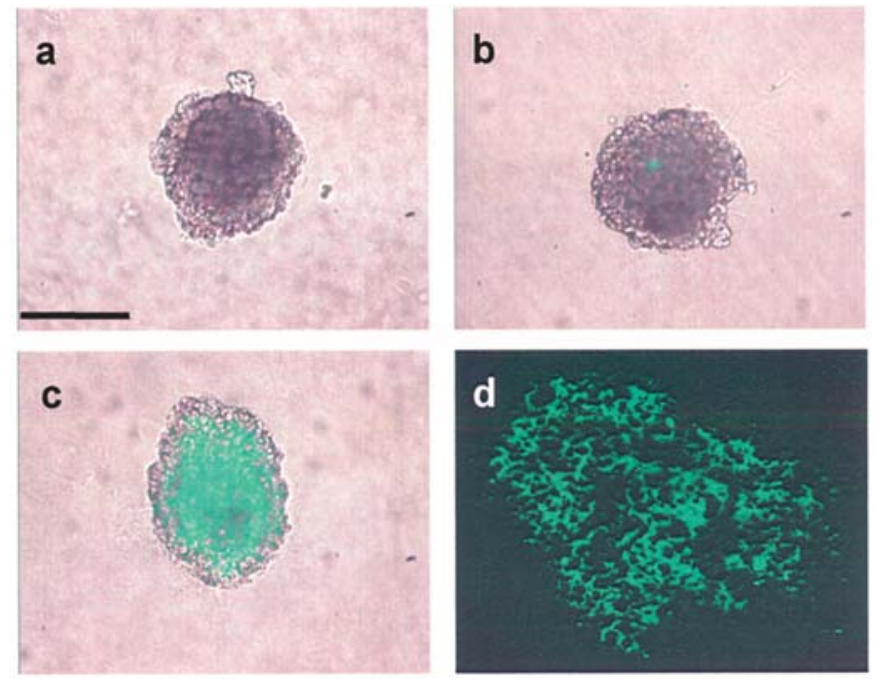

Figure 7. Incorporation of ECM proteins in rBM-induced spheroids of MDA-MB-231 cells. Spheroids were generated from 5000 cells/well with $2.5 \% \mathrm{rBM}$ in the presence of $5 \mu \mathrm{g} / \mathrm{ml}$ rhodamine labeled fibronectin (a), Oregon Green 488-labeled collagen IV (b) and FITC-labeled collagen I (c and d). Overlay of phase contrast and fluorescence image microscopy (a-c) and confocal fluorescence microscopy (d). Cultivation period: $72 \mathrm{~h}$. Bar: $500 \mu \mathrm{m}$.

A decrease of the integrin $\beta 1$ cell surface expression was observed in all cell lines and correlated with the mRNA expression (Fig. 6A, lower panel). An integrin $\beta 1$ surface receptor down-regulation of the majority of the cell population was achieved for MDA-MB-435S, MDA-MB-468 and SKBR-3 (Fig. 6A, lower panel, grey and white bars, and FACS histograms in Fig. 6Be, g and $h$ ) whereas in MCF7, BT474, T-47D, MDA-MB-361 and MDA-MB-231 cells only approximately half of the population displayed a significant decreased level of surface expression after integrin $B 1$ siRNA lipofection (Fig. 6A, lower panel, and FACS histograms in Fig. 6Ba-d and f).

In the spontaneously compact spheroid forming cell lines MCF7, BT-474, T-47D and MDA-MB-361 the downregulation of the integrin $\beta 1$ via siRNA did not alter the spheroid morphology (Fig. 6Ba-d, microscopic images). In
rBM-induced MDA-MB-435S spheroids the down-regulation of integrin $\beta 1$ also did not change the spheroid morphology (Fig. 6Be, microscopic image). In contrast, the rBM-induced breast cancer spheroids of MDA-MB-231 and SK-BR-3 cells were dissociated after integrin $\beta 1$ down-regulation via siRNA (Fig. 6Bf and h, microscopic images). These siRNA findings are in agreement with the inhibitory integrin $\beta 1$ antibody results (Fig. 3). In MDA-MB-468 cells, only a slight morphological disintegration of the spheroid was observed after applying integrin $B 1$ siRNA (Fig. 6Bg, microscopic image).

Collagen I incorporation into rBM-induced spheroids. The role of ECM components in $\mathrm{rBM}$-driven spheroid formation was investigated using the fluorescent matrix proteins collagen I (FITC), collagen IV (Oregon Green 488) and fibronectin (rhodamine) which were added during spheroid formation. As a typical example for Matrigel-inducible breast cancer spheroids we investigated spheroids from the aggregate forming cell line MDA-MB-231. In fully compact MDAMB-231 spheroids only little incorporation of fibronectin and no incorporation of collagen IV into the spheroid was observed (Fig. 7a and b). However, a large amount of collagen I was detected within the spheroid (Fig. 7c). Confocal microscopy reveals that the collagen I molecules assembled into an extracellular meshwork around the cells (Fig. 7d).

\section{Discussion}

A loss or aberrant expression of adhesion molecules has been identified in tumor cells and these alterations favor tumor initiation and progression (20-23). For example, loss of expression or dysfunction of the cell-cell adhesion molecule E-cadherin seems crucial for the development of aggressive, undifferentiated epithelial breast carcinomas $(16,17)$. Expression of $\mathrm{N}$-cadherin, normally restricted to neuronal and mesenchymal cells, has been described in epithelial breast cancer cells and this gain of function is associated with an increased invasive phenotype in breast cancer $(25,26)$. Moreover, an aberrant expression of cellmatrix interacting integrin receptors has been observed in many cancers, enabling anchorage-independent growth, migration

Table II. Adhesion molecule-mediated spheroid formation in eight breast cancer cell lines.

\begin{tabular}{|c|c|c|c|c|c|c|}
\hline Cell line & $\begin{array}{l}\text { Integrin } \beta 1 \\
\text { (siRNA) }\end{array}$ & $(\mathrm{bAB})$ & $\begin{array}{l}\text { E-cadherin } \\
\text { (bAB) }\end{array}$ & $\begin{array}{c}\text { Integrin } \beta 1+\text { E-cadherin } \\
(\mathrm{bAB})\end{array}$ & $\begin{array}{l}\text { N-cadherin } \\
\text { (bAB) }\end{array}$ & Spheroid mediator \\
\hline MCF7 & Spheroid & Spheroid & Aggregate & n.d. & Spheroid & E-cadherin \\
\hline BT-474 & Spheroid & Spheroid & Aggregate & n.d. & Spheroid & E-cadherin \\
\hline $\mathrm{T}-47 \mathrm{D}$ & Spheroid & Spheroid & Aggregate & n.d. & Spheroid & E-cadherin \\
\hline MDA-MB-361 & Spheroid & Spheroid & Aggregate & n.d. & Spheroid & E-cadherin \\
\hline MDA-MB-435S & Spheroid & Spheroid & Spheroid & n.d. & Aggregate & N-cadherin \\
\hline MDA-MB-231 & Aggregate & Aggregate & Spheroid & n.d. & Spheroid & Integrin $ß 1$ \\
\hline MDA-MB-468 & Spheroid & Spheroid & Spheroid & Aggregate & Spheroid & Integrin $\beta 1 /$ E-cadherin \\
\hline SK-BR-3 & Aggregate & Aggregate & Spheroid & n.d. & Spheroid & Integrin $\beta 1$ \\
\hline
\end{tabular}

Adhesion molecules involved in spheroid formation were analyzed by exposing cells either to siRNA or blocking antibody (bAB) prior to 3D spheroid formation. n.d., not determined. 
and metastasis (27-29). In addition, integrin adhesion mediated signaling was shown to contribute to the cell adhesion related drug resistance to chemotherapeutic agents by activating mitogenic pathways and suppressing drug-induced apoptosis (30). For the development and efficacy evaluation of cytotoxic drugs in preclinical settings, the knowledge of drug delivery affecting tumor cell adhesions and recapitulation of these established adhesions in screening assay systems is of utmost importance (12).

The expression of E-cadherin was reported to correlate with a compact spheroid formation in both colon and renal carcinoma cell lines (13-15). On the contrary, for poorly differentiated gastric cancer cells, it was suggested that the formation of tight spheroids was mediated by the loss of E-cadherin expression and upregulation of CD44 adhesion molecule expression (31). We identified that four out of eight breast tumor cell lines formed E-cadherin adhesions and were relevant for spontaneous compact spheroid establishment (Fig. 1a-d), since spheroid formation by these cell lines was abrogated only with antibodies inhibiting the E-cadherin interaction. Neither inhibitory $\mathrm{N}$-cadherin nor integrin $\mathrm{B1}$ antibodies, nor siRNA-mediated down-regulation of the integrin $\beta 1$ receptor subunit interfered with the spheroid formation in these four cell lines. Finally, although low levels of E-cadherin cell surface expression were detectable in MDAMB-468 cells, they did not form spheroids spontaneously. This finding is not unexpected since this cell line is $\alpha$-catenin deficient and the linkage of E-cadherin to the actin cytoskeleton is impaired (32). This indicates that in addition to E-cadherin surface expression, intact intracellular E-cadherin downstream signaling is required for spontaneous spheroid formation. In support of the data reported for colon and renal tumor cells (13-15), our data shows that in breast tumor cells, E-cadherin is the major adhesion molecule mediating tight cell-cell interaction giving rise to true breast tumor spheroids.

In the panel of eight breast cancer cell lines investigated MDA-MB-231, MDA-MB-468 and SK-BR-3 established loose aggregates only (Fig. 1f-h), a morphology that does not coincide with the in vivo xenograft tumors derived from these cell lines. Here, the loss of E-cadherin expression (Table II) might account for this phenotype in vitro. Loss of E-cadherin expression was previously reported in MDA-MB-231 by promoter hypermethylation (33), in SK-BR-3 by homozygous deletion of large parts of the gene (34) and a defect in signaling transmission in MDA-MB-468 was described (32). However, in the presence of ECM proteins these cell lines adopt a spheroid morphology (Fig. 1f'-h'). This indicates that in contrast to the E- and N-cadherin-mediated spontaneous formation of spheroids, the compaction of aggregates into spheroids is mediated exclusively by integrin ß1-ECM interaction. It is tempting to speculate that this interaction might confer tumor tightness in vivo in E-cadherin-defective tumor cells.

A recent report indicates that the inhibition of integrin $\beta 1$ subunit induces proliferation arrest and apoptosis in breast cancer cell lines grown in 3D in a laminin-rich extracellular basement matrix (35). However, in our experimental 3D setting the inhibition of integrin $\beta 1$ receptor did not induce cell death but spheroid disintegration only (Fig. 5c"). This discrepancy of the biological effect of integrin $\beta 1$ inhibition might be explained by the different functional properties and concentrations of the two integrin $\beta 1$ blocking antibodies used. Our spheroid disintegration was observed with an anti-integrin $\beta 1$ antibody clone P5D2 at $10 \mu \mathrm{g} / \mathrm{ml}$, whereas the apoptosis detection was observed after incubation of the cells with anti-integrin antibody clone AIIB2, applying a rather high concentration of $80-240 \mu \mathrm{g} / \mathrm{ml}$ (35). An alternative explanation might be the use of different culture settings of laminin and reconstituted basement membrane supplements. This is supported by the fact that opposing cell death effects were reported from the same group using the integrin $\beta 1$ blocking antibody clone AIIB2 in MCF7 spheroids $(35,36)$. In laminin rich rBM, blockage of the integrin $B 1$ subunit induced apoptosis in MCF7 cells (35), whereas neither growth arrest nor apoptosis was found in Matrigel ${ }^{\mathrm{TM}}$ or collagen I supplemented medium (36). Nevertheless, our data indicate that the disintegration of $3 \mathrm{D}$ tumor spheroids solely does not induce cell death in breast cancer cells.

Expression and gain of function of $\mathrm{N}$-cadherin has been described in many invasive breast cancer cell lines $(25,26)$. Our investigations revealed that the $\mathrm{N}$-cadherin-positive breast tumor cell line MDA-MB-435S develops a tight aggregate morphology in 3D and establishes a higher degree of compaction when supplemented with ECM proteins (Fig. 1e'). Since in MDA-MB-435S cells an inhibitory $\mathrm{N}$-cadherin antibody dissociated the spheroid into a loose aggregate (Fig. 4Bc), this suggests that the initial tight aggregation of cells is mediated by the N-cadherin homophilic interactions whereas additional tightness is conferred via ECM protein/cellular receptor interactions. The exceptional behavior of MDA-MB-435S cells might relate to the discussed melanoma origin of this cell line (37).

A more complex cell adhesion interaction pattern exists in moderate E-cadherin-positive MDA-MB-468 cells. The 3D morphology could be transformed from aggregates into tight spheroids by the addition of $\mathrm{rBM}$, indicative of the fact that integrins are involved in cell adhesion to generate spheroids (Fig. 1g). These spheroids could not be dissociated solely by inhibition of integrin $\beta 1$, but only by a combination of Ecadherin and integrin $\beta 1$ blocking antibodies (Fig. 3g and 4Ac). This indicates that in MDA-MB-468 cells, ECM proteinreceptor interactions activate integrin-mediated adhesion pathways which in turn might stimulate E-cadherin-mediated intercellular adhesion in this cell model. This suggestion is in line with the previously reported crosstalk of the E-cadherin cell-cell adhesion with integrin (38) and growth factor receptor pathways (39).

We described recently that collagen I as a single agent was capable to induce some degree of compaction in $3 \mathrm{D}$ aggregates. However, complete compaction was achieved only after medium supplementation with rBM (11). As shown in Fig. 7, in the presence of rBM, fluorescent collagen I molecules are located around the cells forming a network that interconnects and compacts cells to spheroids. This finding further supports the suggestion of collagen I as a major matrix molecule involved in the spheroid compaction in breast cancer cells. Interestingly, collagen I derived from $\mathrm{rBM}$ is superior to the pure collagen I preparation in terms of spheroid compaction (11) which might be due to the presence of fibrillar collagen I in rBM which can evolve into a branched 
meshwork connecting adjacent cells in the spheroid. Collagen IV and fibronectin seem not to be involved in the rBM-induced spheroid compaction in breast cancer cells (Fig. 7).

In summary, our findings indicate that in more dedifferentiated breast cancer cells integrin-mediated cell-matrix interactions might be the dominant cell-cell interaction tightness determining factor, increasing the cellular flexibility to evolve into a more invasive phenotype.

A diversity of different cadherin and integrin adhesion receptors contribute either as a single target or in combination to the formation of true $3 \mathrm{D}$ spheroids in vitro. Although integrin $\beta 1$ is expressed in all breast tumor models investigated, its importance for tight cell packaging becomes evident only in the more dedifferentiated E-cadherin-negative tumor cell lines. Since the majority of breast tumor cell lines display a loss of E-cadherin function (40), their 3D tumor cultures are dominated by the formation of aggregates. These aggregates are no longer useful as in vitro counterparts of avascular tumors of in vivo xenografts, since they lack diffusion barriers (no nutrient limitation) as well as a stratified cellular composition with proliferating cells in the rim and quiescent and dead cells in the core. However, in the presence of matrix components, the cellular interactions via integrin $\beta 1$ receptors compensate the loss of cadherins leading to the formation of true spheroids, making these cadherin-negative breast cancer spheroids useful models to study the performance of anti-tumor drugs. Moreover, the understanding of adhesion receptor expression patterns is important for the development and evaluation of new anti-tumor therapies since adhesion molecules are themselves targets for anti-cancer therapy (41-43) and adhesion receptors might be targets to sensitize tumor cells to therapeutic antibodies or host immune-mediated toxicity (44).

\section{References}

1. Kunz-Schughart LA, Freyer JP, Hofstaedter F and Ebner R: The use of 3-D cultures for high-throughput screening: the multicellular spheroid model. J Biomol Screen 9: 273-285, 2004.

2. Minchinton AI and Tannock IF: Drug penetration in solid tumours. Nat Rev Cancer 6: 583-592, 2006.

3. Kim JB, Stein R and O'Hare MJ: Three-dimensional in vitro tissue culture models of breast cancer - a review. Breast Cancer Res Treat 85: 281-291, 2004.

4. Shaw KR, Wrobel CN and Brugge JS: Use of three-dimensional basement membrane cultures to model oncogene-induced changes in mammary epithelial morphogenesis. J Mammary Gland Biol Neoplasia 9: 297-310, 2004.

5. Desoize B, Gimonet D and Jardiller JC: Cell culture as spheroids: an approach to multicellular resistance. Anticancer Res 18: 4147-4158, 1998.

6. Mueller-Klieser W: Tumor biology and experimental therapeutics. Crit Rev Oncol Hematol 36: 123-139, 2000.

7. Bates RC, Edwards NS and Yates JD: Spheroids and cell survival. Crit Rev Oncol Hematol 36: 61-74, 2000.

8. Sutherland RM: Cell and environment interactions in tumor microregions: the multicell spheroid model. Science 240: 177$184,1988$.

9. St Croix B, Man S and Kerbel RS: Reversal of intrinsic and acquired forms of drug resistance by hyaluronidase treatment of solid tumors. Cancer Lett 131: 35-44, 1998.

10. Green SK, Francia G, Isidoro C and Kerbel RS: Antiadhesive antibodies targeting E-cadherin sensitize multicellular tumor spheroids to chemotherapy in vitro. Mol Cancer Ther 3: 149-159, 2004.

11. Ivascu A and Kubbies M: Rapid generation of single-tumor spheroids for high-throughput cell function and toxicity analysis. J Biomol Screen 11: 922-932, 2006.
12. Grantab R, Sivananthan S and Tannock IF: The penetration of anticancer drugs through tumor tissue as a function of cellular adhesion and packing density of tumor cells. Cancer Res 66: 1033-1039, 2006.

13. Shimazui T, Schalken JA, Kawai K, Kawamoto R, van Bockhoven A, Oosterwijk E and Akaza H: Role of complex cadherins in cell-cell adhesion evaluated by spheroid formation in renal cell carcinoma cell lines. Oncol Rep 11: 357-360, 2004.

14. St Croix B, Sheehan C, Rak JW, Florenes VA, Slingerland JM and Kerbel RS: E-Cadherin-dependent growth suppression is mediated by the cyclin-dependent kinase inhibitor p27(KIP1). J Cell Biol 142: 557-571, 1998.

15. Shimoyama Y, Nagafuchi A, Fujita S, Gotoh M, Takeichi M, Tsukita S and Hirohashi S: Cadherin dysfunction in a human cancer cell line: possible involvement of loss of alpha-catenin expression in reduced cell-cell adhesiveness. Cancer Res 52: 5770-5774, 1992

16. Berx G, Cleton-Jansen AM, Nollet F, de Leeuw WJ, van de Vijver M, Cornelisse C and van Roy F: E-cadherin is a tumour/ invasion suppressor gene mutated in human lobular breast cancers. EMBO J 14: 6107-6115, 1995.

17. Moll R, Mitze M, Frixen UH and Birchmeier W: Differential loss of E-cadherin expression in infiltrating ductal and lobular breast carcinomas. Am J Pathol 143: 1731-1742, 1993.

18. Casey RC, Burleson KM, Skubitz KM, Pambuccian SE, Oegema TR Jr, Ruff LE and Skubitz AP: Beta 1-integrins regulate the formation and adhesion of ovarian carcinoma multicellular spheroids. Am J Pathol 159: 2071-2080, 2001.

19. Robinson EE, Zazzali KM, Corbett SA and Foty RA: Alpha5beta1 integrin mediates strong tissue cohesion. J Cell Sci 116: $377-$ 386, 2003.

20. Foty RA and Steinberg MS: Cadherin-mediated cell-cell adhesion and tissue segregation in relation to malignancy. Int J Dev Biol 48: 397-409, 2004.

21. Derycke LD and Bracke ME: N-cadherin in the spotlight of cell-cell adhesion, differentiation, embryogenesis, invasion and signalling. Int J Dev Biol 48: 463-476, 2004.

22. Guo W and Giancotti FG: Integrin signalling during tumour progression. Nat Rev Mol Cell Biol 5: 816-826, 2004.

23. Hazan RB, Qiao R, Keren R, Badano I and Suyama K: Cadherin switch in tumor progression. Ann NY Acad Sci 14: 155-163, 2004

24. Hoevel T, Macek R, Swisshelm K and Kubbies M: Reexpression of the TJ protein CLDN1 induces apoptosis in breast tumor spheroids. Int J Cancer 108: 374-383, 2004.

25. Nieman MT, Prudoff RS, Johnson KR and Wheelock MJ: $\mathrm{N}$-cadherin promotes motility in human breast cancer cells regardless of their E-cadherin expression. J Cell Biol 147: 631$644,1999$.

26. Hazan RB, Phillips GR, Qiao RF, Norton L and Aaronson SA: Exogenous expression of $\mathrm{N}$-cadherin in breast cancer cells induces cell migration, invasion, and metastasis. J Cell Biol 148: 779-790, 2000.

27. Felding-Habermann B, O'Toole TE, Smith JW, Fransvea E, Ruggeri ZM, Ginsberg MH, Hughes PE, Pampori N, Shattil SJ, Saven A and Mueller BM: Integrin activation controls metastasis in human breast cancer. Proc Natl Acad Sci USA 98: 1853-1858, 2001.

28. Morini M, Mottolese M, Ferrari N, Ghiorzo F, Buglioni S, Mortarini R, Noonan DM, Natali PG and Albini A: The alpha 3 beta 1 integrin is associated with mammary carcinoma cell metastasis, invasion, and gelatinase B (MMP-9) activity. Int J Cancer 87: 336-342, 2000.

29. Wewer UM, Shaw LM, Albrechtsen R and Mercurio AM: The integrin alpha 6 beta 1 promotes the survival of metastatic human breast carcinoma cells in mice. Am J Pathol 151: 1191-1198, 1997.

30. Bewick MA and Lafrenie RM: Adhesion dependent signalling in the tumour microenvironment: the future of drug targetting. Curr Pharm Res 12: 2833-2848, 2006.

31. Mayer B, Klement G, Kaneko M, Man S, Jothy S, Rak J and Kerbel RS: Multicellular gastric cancer spheroids recapitulate growth pattern and differentiation phenotype of human gastric carcinomas. Gastroenterology 121: 839-852, 2001.

32. Pierceall WE, Woodard AS, Morrow JS, Rimm D and Fearon ER: Frequent alterations in E-cadherin and alpha- and beta-catenin expression in human breast cancer cell lines. Oncogene 11: 1319-1326, 1995.

33. Graff JR, Herman JG, Lapidus RG, Chopra H, Xu R, Jarrard DF, Isaacs WB, Pitha PM, Davidson NE and Baylin SB: E-cadherin expression is silenced by DNA hypermethylation in human breast and prostate carcinomas. Cancer Res 55: 5195-5159, 1995. 
34. van de Wetering M, Barker N, Harkes IC, van der Heyden M, Dijk NJ, Hollestelle A, Klijn JG, Clevers $\mathrm{H}$ and Schutte M: Mutant E-cadherin breast cancer cells do not display constitutive Wnt signaling. Cancer Res 61: 278-284, 2001.

35. Park CC, Zhang H, Pallavicini M, Gray JW, Baehner F, Park CJ and Bissell MJ: Beta1 integrin inhibitory antibody induces apoptosis of breast cancer cells, inhibits growth, and distinguishes malignant from normal phenotype in three dimensional cultures and in vivo. Cancer Res 66: 1526-1535, 2006.

36. Howlett AR, Bailey N, Damsky C, Petersen OW and Bissell MJ: Cellular growth and survival are mediated by beta 1 integrins in normal human breast epithelium but not in breast carcinoma. J Cell Sci 108: 1945-1957, 1995.

37. Rae JM, Ramus SJ, Waltham M, Armes JE, Campbell IG, Clarke R, Barndt RJ, Johnson MD and Thompson EW: Common origins of MDA-MB-435 cells from various sources with those shown to have melanoma properties. Clin Exp Metastasis 21: 543-552, 2004.

38. von Schlippe M, Marshall JF, Perry P, Stone M, Zhu AJ and Hart IR: Functional interaction between E-cadherin and alphavcontaining integrins in carcinoma cells. J Cell Sci 113: 425-437, 2000.

39. Hazan RB and Norton L: The epidermal growth factor receptor modulates the interaction of E-cadherin with the actin cytoskeleton. J Biol Chem 273: 9078-9084, 1998.
40. Sommers CL, Byers SW, Thompson EW, Torri JA and Gelmann EP: Differentiation state and invasiveness of human breast cancer cell lines. Breast Cancer Res Treat 31: 325-335, 1994.

41. Trikha M, Zhou Z, Nemeth JA, Chen Q, Sharp C, Emmell E, Giles-Komar J and Nakada MT: CNTO 95, a fully human monoclonal antibody that inhibits alphav integrins, has antitumor and antiangiogenic activity in vivo. Int J Cancer 110: 326-335, 2004.

42. Eskens FA, Dumez H, Hoekstra R, Perschl A, Brindley C, Bottcher S, Wynendaele W, Drevs J, Verweij J and van Oosterom AT: Phase I and pharmacokinetic study of continuous twice weekly intravenous administration of Cilengitide (EMD 121974), a novel inhibitor of the integrins alphavbeta3 and alphavbeta5 in patients with advanced solid tumours. Eur J Cancer 39: 917-926, 2003.

43. Burke PA, DeNardo SJ, Miers LA, Lamborn KR, Matzku S and DeNardo GL: Cilengitide targeting of alpha(v)beta(3) integrin receptor synergizes with radioimmunotherapy to increase efficacy and apoptosis in breast cancer xenografts. Cancer Res 62: 4263-4272, 2002.

44. Green SK, Karlsson MC, Ravetch JV and Kerbel RS: Disruption of cell-cell adhesion enhances antibody-dependent cellular cytotoxicity: implications for antibody-based therapeutics of cancer. Cancer Res 62: 6891-6900, 2002. 